\title{
Implementation of non-local xor function with coherent-state qubit
}

\author{
João Batista Rosa Silva and Rubens Viana Ramos
}

\begin{abstract}
This work describes how to implement a non-local xor function with coherent-state qubit using only linear optics devices and having efficiency equal to $1 / 2$. The key element that makes possible the realization of the non-local xor function is a tripartite GHZ-type entangled coherent state. Its generation is proposed using an ideal lossless setup.
\end{abstract}

Keywords - Quantum teleportation, coherent state qubit, tripartite states.

\section{INTRODUCTION}

Optical systems is one of the most promising technologies that can bring closer the implementation and use of quantum communication protocols and quantum computing. Even inside of optical technologies, there are different possibilities for the qubit implementation, being the singlephoton polarization, phase and time-bin the most commonly qubit implementations used [1-5]. Their advantages are the facility to produce entangled states through parametric down conversion, construction of probabilistic CNOT with common optical devices and the easy implementation of single-qubit gates. The disadvantage of such qubit implementations is the fact that quantum information is carried by a single-photon, this makes the system very sensitive to losses and it requires good single-photon detectors. On the other hand the qubit implementation using superposition of coherent states has been proposed [6-9]. Such qubit implementation has as advantages the fact that it does not need single-photon detectors and the losses in the optical devices cause a quantum error (that can be corrected by a quantum code) but not destruction of the quantum information. Its disadvantages are the hard production of coherent state superposition [10-12] and the fact that the implementation of single-qubit gates requires the teleportation procedure and photon number resolution. In this work, we propose an optical setup for implementation of the non-local xor function for coherent state quantum information processing (CSQIP), including the optical setup for generation of the entangled state required by the protocol.

Before starting the main work of this paper, we give a short review of CSQIP. Coherent states are eigenstates of the annihilation operator $\hat{a}$, with complex eigenvalue $\alpha$, i.e. $\hat{a}|\alpha\rangle=\alpha|\alpha\rangle$. In CSQIP, the qubit is encoded as $\left|0_{L}\right\rangle=|-\alpha\rangle$ and

\footnotetext{
${ }^{1}$ João Batista Rosa Silva and ${ }^{2}$ Rubens Viana Ramos are with ${ }^{1,2}$ Department of Teleinformatic Engineering, Federal University of Ceara (UFC) and ${ }^{1}$ Center of Technological Sciences - Telecommunications Engineering University of Fortaleza (UNIFOR), Fortaleza, Ceará, Brazil, emails: joaobrs@[deti.ufc.br, unifor.br], rubens@deti.ufc.br.
}

$\left|1_{L}\right\rangle=|\alpha\rangle$ where $\alpha$ is assumed to be real. In this case, one has $\left|\left\langle 0_{L} \mid 1_{L}\right\rangle\right|^{2}=|\langle-\alpha \mid \alpha\rangle|^{2}=\exp \left(-4|\alpha|^{2}\right)$. Most of gates in CSQIP requires $\alpha \geq 2$, thus $|\langle\alpha \mid-\alpha\rangle|^{2} \leq 1.11254 \cdot 10^{-7}$, which gives a good approximation for the orthogonality. The main optical devices used in the implementation of CSQIP are the beam splitter (BS) and the phase modulator (PM). The unitary operator of a lossless BS is $B=\exp \left[\pi\left(\hat{a}_{1} \hat{a}_{2}^{\dagger}-\hat{a}_{1}^{\dagger} \hat{a}_{2}\right) / 4\right]$. Thus, when two coherent states $|\alpha\rangle_{1}$ and $|\beta\rangle_{2}$ enter at the input ports of a BS, the total state at the output ports is

$$
|\alpha, \beta\rangle_{1,2} \stackrel{B S}{\longrightarrow}|(\alpha-\beta) / \sqrt{2},(\alpha+\beta) / \sqrt{2}\rangle_{1,2} \text {. }
$$

From (1), if $\beta=\alpha(\beta=-\alpha)$, the vacuum state appears at output mode 1(2). Hence, the setup for qubit measurement in the canonical basis consist of a BS, two common photodetectors (placed at the output ports of the BS) and a local oscillator in the state $|\alpha\rangle$. The logical state of the measured qubit is defined according to in which detector photons were received. The PM, by its turn, adds a phase $\theta$ to the signal that passes through it. Its unitary operator is $\hat{U}(\theta)=\exp \left(i \theta \hat{a}^{\dagger} \hat{a}\right)$ and it acts like

$$
|\alpha\rangle \stackrel{P M}{\longrightarrow}|\exp (i \theta) \alpha\rangle
$$

Thus, if $\theta=\pi$, and the light passing by the PM is a coherent state $|\alpha\rangle(-|\alpha\rangle)$, then the output state will be $|-\alpha\rangle(|\alpha\rangle)$, thus, the PM with $\theta=\pi$ is a NOT gate in CSQIP.

The rest of this work is outlined as follows: Section II begins with a review of the teleportation of the xor function, after, the optical implementation of the non-local XOR function using only linear optical devices is presented; at last, the conclusions are presented in Section III.

\section{Teleportation of the Xor Function Between Two Classcial Bits}

The quantum teleportation of the xor function between two classical bits was proposed firstly in [13] and it can be used in several protocols as quantum key distribution, error correction, control of channel access, among others. The main element of this protocol is tripartite GHZ-type state. Thus, we initially consider that there are three authorized parties of the communication, Alice, Bob and Charlie, sharing the following maximally entangled tripartite of qubit state: 


$$
|\psi\rangle=\frac{1}{2}\left(|000\rangle_{A B C}+|011\rangle_{A B C}+|110\rangle_{A B C}+|101\rangle_{A B C}\right)
$$

Considering $\rho_{A}, \rho_{B}$ and $\rho_{C}$ as the individual parts of the total state $|\psi\rangle$, the teleportation of the xor function between two classical bits, represented by $K$ (belonging to Alice) and $R$ (belonging to Bob) can be achieved using the quantum circuit shown in Fig. 1.

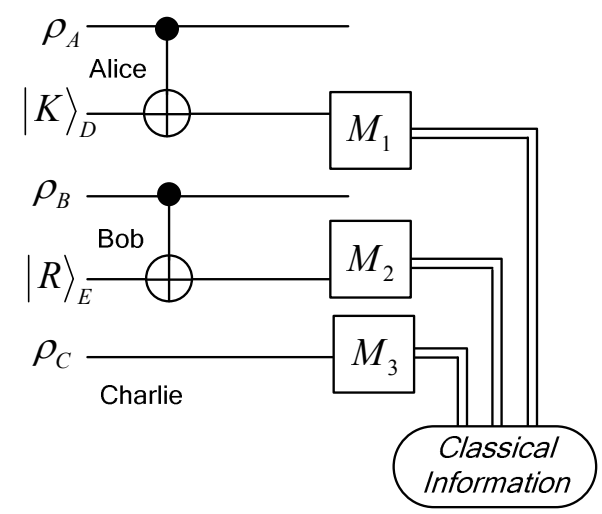

Fig. 1. Quantum circuit for teleportation of the xor function between two classical bits. $M_{1-3}$ are qubit measurers.

In Fig. $1, M_{1}, M_{2}$ and $M_{3}$ are measurers The initial and final states are, respectively, given by:

$$
\begin{gathered}
\left|\Psi_{\text {in }}\right\rangle=|K R\rangle_{D E} \otimes \frac{1}{2}\left(|000\rangle_{A B C}+|011\rangle_{A B C}+|110\rangle_{A B C}+|101\rangle_{A B C}\right) \\
\left|\Psi_{\text {out }}\right\rangle=\frac{1}{2}\left\{\begin{array}{l}
|00\rangle_{A B}|K R\rangle_{D E}|0\rangle_{C}+|01\rangle_{A B}|K \bar{R}\rangle_{D E}|1\rangle_{C}+ \\
|11\rangle_{A B}|\bar{K} \bar{R}\rangle_{D E}|0\rangle_{C}+|10\rangle_{A B}|\bar{K} R\rangle_{D E}|1\rangle_{C}
\end{array}\right\}
\end{gathered}
$$

In (5), when the qubits $D, E$ and $C$ are measured, by Alice, Bob and Charlie, respectively, the values $\{110,101,000$, $011\}_{\text {DEC }}$ are obtained only if bits $K$ and $R$ are equal. On the other hand, if $K$ and $R$ are not equal only the values $\{100$, $111,010,001\}_{\mathrm{DEC}}$ can be obtained in the measurements. Hence, the protocol of quantum teleportation of the xor function between two classical bits can be described as follows:

- Alice performs a measurement in the qubit $D$ and she sends her result to Charlie using one classical bit.

- Bob performs a measurement in the qubit $E$ and he sends his result to Charlie using another classical bit.

- Charlie, by its turn, performs a measurement in his qubit. Knowing those three classical information, Charlie can know if $K$ and $R$ are equal or not. Hence, the xor function between the classical bits belonging to Alice and Bob is teleported to Charlie.
The classical bits sent by Alice and Bob inform to Charlie not the values of $K$ and $R$, but if $K$ and $R$ are equal or not to the individual states $\rho_{A}$ and $\rho_{B}$, respectively.

As seen before, in order to realize the teleportation of the xor function between two classical bits, the first task to be done is the generation of the tripartite entangled state (3). In CSQIP, the state (3) is rewritten as

$$
|\psi\rangle=2 N^{4}(|-\alpha,-\alpha,-\alpha\rangle+|-\alpha, \alpha, \alpha\rangle+|\alpha,-\alpha, \alpha\rangle+|\alpha, \alpha,-\alpha\rangle),
$$

where $N=\left[2\left(1+e^{-2 \alpha^{2}}\right)\right]^{-1 / 2}$ is the normalization constant. The state (6) can be obtained by application of a Hadamard gate in each individual state of the tripartite GHZ state $(|-\alpha,-\alpha,-\alpha\rangle+|\alpha, \alpha, \alpha\rangle) / 2^{1 / 2}$. This last one can be generated by the optical setup proposed in $[6,14]$ and shown in Fig. 2, where the beam splitters BS1 and BS2 have, respectively, reflectivity equal to $3^{-1 / 2}$ and $2^{-1 / 2}$.

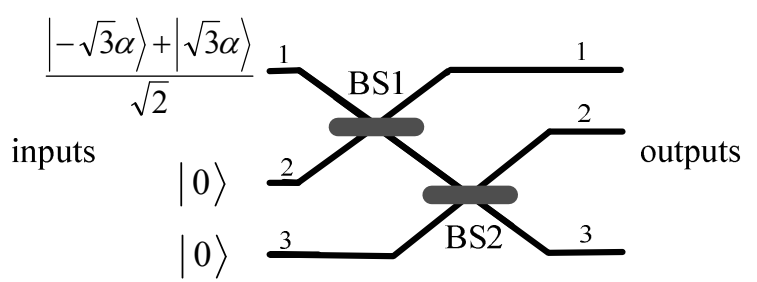

Fig. 2. Entanglement generator circuit for GHZ state $(\mid-\alpha,-\alpha$, $\alpha\rangle+|\alpha, \alpha, \alpha\rangle) / 2^{1 / 2}$.

The Hadamard gate in coherent state encoding has to realize the transformations $|-\alpha\rangle \rightarrow(|-\alpha\rangle+|\alpha\rangle) / 2^{1 / 2}$ and $|\alpha\rangle \rightarrow(|-\alpha\rangle$ $|\alpha\rangle) / 2^{1 / 2}$. This operation takes non-orthogonal states to orthogonal states and, hence, it is not unitary. The implementation of a Hadamard gate in CSQIP requires the realization of a teleportation and it succeeds with probability (per turn) depending on $\alpha$ [6]. Thus, the efficiency of generation of the state given in (6) using the setup shown in Fig. 2 [14] and three Hadamard gates proposed in [6] is not very efficient. Thus, we propose the setup in Fig. 3 for generation of the state in (6). In Fig. 3, $|\xi\rangle=N(|-\alpha\rangle+|\alpha\rangle) / 2^{1 / 2}$. After some trivial calculations, one can find the following output state $\left|\Psi_{\mathrm{o}}\right\rangle$ before the homodyne detection

$$
\begin{aligned}
& \left|\Psi_{o}\right\rangle=N\left[N\left(\left|\psi_{1}\right\rangle+\left|\psi_{2}\right\rangle\right)+\left|\psi_{u}\right\rangle\right] \\
& \left|\psi_{1}\right\rangle=N^{2}\left(\begin{array}{l}
|\alpha,-\alpha,-\alpha,-\alpha\rangle+|\alpha,-\alpha, \alpha, \alpha\rangle+ \\
|\alpha, \alpha,-\alpha, \alpha\rangle+|\alpha, \alpha, \alpha,-\alpha\rangle
\end{array}\right)_{1-4}=|\alpha\rangle_{1}|\psi\rangle_{234} \\
& \left|\psi_{2}\right\rangle=(I \otimes I \otimes I \otimes X)|-\alpha\rangle_{1}|\psi\rangle_{234} \\
& \left|\psi_{u}\right\rangle=N^{3}\left(\begin{array}{l}
|\varnothing, \varnothing,-2 \alpha, \varnothing\rangle+|\varnothing,-2 \alpha, \varnothing, \varnothing\rangle+ \\
|\varnothing, \varnothing, \varnothing,-2 \alpha\rangle+|-2 \alpha, \varnothing, \varnothing, \varnothing\rangle+ \\
|2 \alpha, \varnothing, \varnothing, \varnothing\rangle+|\varnothing, \varnothing, \varnothing, 2 \alpha\rangle+ \\
|\varnothing, 2 \alpha, \varnothing, \varnothing\rangle+|\varnothing, \varnothing, 2 \alpha, \varnothing\rangle
\end{array}\right)_{1-4}
\end{aligned}
$$




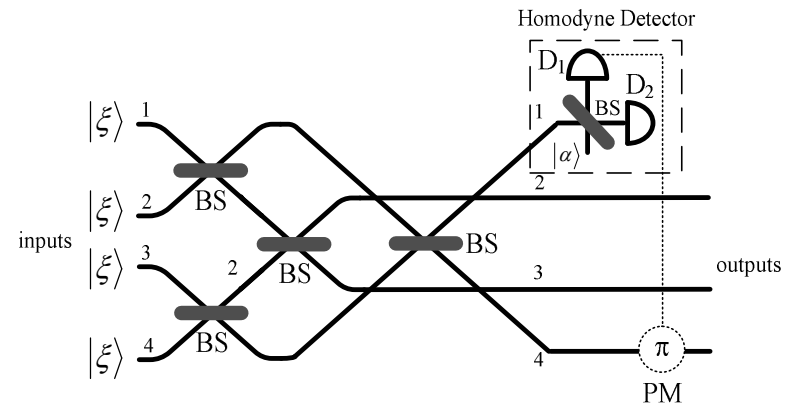

Fig. 3. Setup for generation of the tripartite entangled state given in (6).

In (7), $\left|\Psi_{u}\right\rangle$ is the useless part that contains the situations where detection happen in both or none detectors, $D_{1}$ and $\mathrm{D}_{2}$, in this case, the circuit fails $(|\varnothing\rangle$ is the vacuum state). From (7) one can also note that when the homodyne detector measures $|\alpha\rangle$, the output is $|\psi\rangle$ and the $X$ gate (PM) is disabled. On the other hand, if the result of the measurement is $|-\alpha\rangle$, then the $X$ gate is activated in order to correct the output state, according to (9). The probability of success of the setup shown in Fig. 3 is about $1 / 2$ for $\alpha \geq 2$.

Once the required tripartite state was generated, one can use the setup in Fig. 4 to implement the quantum circuit presented in Fig. 1 and run the non-local xor function protocol.

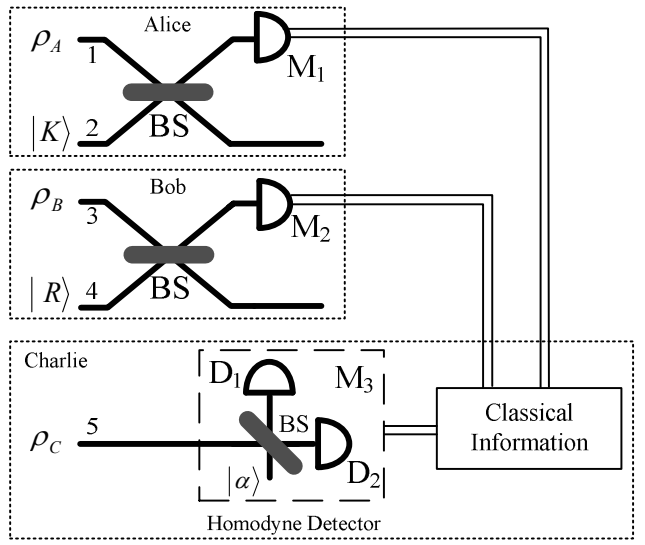

Fig. 4. Optical setup for teleportation of the xor function of two classical bits.

The input state is $\left[2^{-1}(|-\alpha,-\alpha, \alpha\rangle+|-\alpha, \alpha, \alpha\rangle+|\alpha,-\alpha, \alpha\rangle+\mid \alpha, \alpha,-\right.$ $\left.\alpha\rangle)_{135}\right] \otimes|K\rangle_{2} \otimes|R\rangle_{4}$, where $K$ and $R \in\{-\alpha, \alpha\}$. The total quantum state just before the measurements is

$$
\left|\Psi_{o}\right\rangle=\frac{1}{2}\left(\begin{array}{l}
\left|\frac{-(\alpha+K)}{\sqrt{2}}, \frac{K-\alpha}{\sqrt{2}}, \frac{-(\alpha+R)}{\sqrt{2}}, \frac{R-\alpha}{\sqrt{2}},-\alpha\right\rangle+ \\
\left|\frac{-(\alpha+K)}{\sqrt{2}}, \frac{K-\alpha}{\sqrt{2}}, \frac{\alpha-R}{\sqrt{2}}, \frac{R+\alpha}{\sqrt{2}}, \alpha\right\rangle+ \\
\left|\frac{\alpha-K}{\sqrt{2}}, \frac{K+\alpha}{\sqrt{2}}, \frac{-(\alpha+R)}{\sqrt{2}}, \frac{R-\alpha}{\sqrt{2}}, \alpha\right\rangle+ \\
\left|\frac{\alpha-K}{\sqrt{2}}, \frac{K+\alpha}{\sqrt{2}}, \frac{\alpha-R}{\sqrt{2}}, \frac{R+\alpha}{\sqrt{2}},-\alpha\right\rangle
\end{array}\right)_{12345}^{(11)}
$$

The measurers $M_{1}$ and $M_{2}$ just detect the presence or absence of the light meaning, respectively, bit ' 1 ' and bit ' 0 '. Charlie uses a measurer based in homodyne detection. Therefore, when the qubits $A, B$ and $C$ are measured, by Alice, Bob and Charlie, respectively, the values $\{110,101$, $000,011\}_{A B C}$ are obtained only if qubits $K$ and $R$ are equal. On the other hand, if $K$ and $R$ are not equal only the values $\{100,111,010,001\}_{A B C}$ can be obtained by the measurement conform described by the quantum teleportation protocol of the xor function between two classical bits.

\section{CONCLUSIONS}

Firstly, we proposed a setup for probabilistic generation of the tripartite GHZ state (6) that it is used to realize the quantum teleportation protocol of the xor function between two classical bits. Its success probability is of $1 / 2$. In the sequence, we presented a proposal of realization of the quantum teleportation protocol of the xor function using only linear optical devices with coherent-state qubit. The efficiency of the teleporter circuit of the xor function is 1 . Thus, the total efficiency is $1 / 2$.

\section{REFERENCES}

[1] T. C. Ralph, A. G. White, W. J. Munro, and G. J. Milburn, "Simple scheme for efficient linear optics quantum gates", Phys. Rev. A, 65, pp. $012314,2001$.

[2] T. B. Pittman, B. C. Jacobs, and J. D. Franson, "Probabilistic quantum logic operations using polarizing beam splitters", Phys. Rev. A, 64, pp. 062311/1-9, 2001.

[3] T. B. Pittman, M. J. Fitch, B. C. Jacobs, and J. D. Franson, "Experimental controlled-NOT logic gate for single photons in the coincidence basis", Phys. Rev. A, 68, pp. 032316/1-4, 2003.

[4] S. J. D. Phoenix and P. D. Townsend, "Quantum cryptography: how to beat the code breakers using quantum mechanics", Contemp. Phys., 36 pp. 165-195, 1995.

[5] N. Gisin, G. Ribordy, W. Tittel and H. Zbinden, "Quantum cryptography", Los Alamos e-print quant-ph/0101098, 2001.

[6] S. Glancy, H. Vasconcelos and T. C. Ralph, "Transmission of optical coherent-state qubits", Phys. Rev. A, 70, pp. 22317, 2004

[7] H. Jeong and M. Kim, "Efficient quantum computation using coherent states", Phys. Rev. A, 65, pp. 042305, 2002.

[8] T. C. Ralph, A. Gilchrist, G. Milburn, W. Munro and S. Glancy, "Quantum computation with optical coherent states",Phys. Rev. A, 68, pp. 42319, 2003. 
[9] H. H. M. de Vasconcelos. Topics in coherent state quantum computation and state purification. Doctor of philosophy thesis, Graduate Program in Physics Notre Dame, Indiana July 2006.

[10] H. Jeong, M. Kim, T. Ralph and B. Ham, "Generation of macroscopic superposition states with small nonlinearity” Phys. Rev. A, 70, 61801, 2004.

[11] A. P. Lund, H. Jeong, T. C. Ralph and M. S. Kim, "Conditional production of superpositions of coherent states with inefficient photon detection", Phys. Rev. A, 70, pp. 20101, 2004.

[12] H. Jeong, A. P. Lund and T. C. Ralph, "Production of superpositions of coherent states in traveling optical fields with inefficient photon detection", Phys. Rev A, 72, pp. 13801, 2005.

[13] P. B. M. de Sousa, J. B. R. Silva and R. V. Ramos, "Implementing non-local xor function with quantum communication", J. of Mod. Opt., 53, 12, pp. 1765, 2006

[14] H. Jeong and N. Ba An, "Greenberg-Horn-Zeilinger-type and W-type entangled coherent states: generation and Bell-type inequality tests without photon counting", Phys. Rev. A, 74, pp. 022104, 2006. 